PROCEEDINGS OF THE

AMERICAN MATHEMATICAL SOCIETY

Volume 128, Number 7, Pages 1885-1892

S 0002-9939(99)05306-X

Article electronically published on November 1, 1999

\title{
SCHREIER THEOREM ON GROUPS WHICH SPLIT OVER FREE ABELIAN GROUPS
}

\author{
MYOUNGHO MOON
}

(Communicated by Ralph Cohen)

\begin{abstract}
Let $G$ be either a free product with amalgamation $A *_{C} B$ or an HNN group $A *_{C}$, where $C$ is isomorphic to a free abelian group of finite rank. Suppose that both $A$ and $B$ have no nontrivial, finitely generated, normal subgroups of infinite indices. We show that if $G$ contains a finitely generated normal subgroup $N$ which is neither contained in $C$ nor free, then the index of $N$ in $G$ is finite. Further, as an application of this result, we show that the fundamental group of a torus sum of 3-manifolds $M_{1}$ and $M_{2}$, the interiors of which admit hyperbolic structures, have no nontrivial, finitely generated, nonfree, normal subgroup of infinite index if each of $M_{1}$ and $M_{2}$ has at least one nontorus boundary.
\end{abstract}

\section{INTRODUCTION}

Some classes of groups are known to have no nontrivial, finitely generated, normal subgroup of infinite index. Let $\mathcal{S}$ be the class of groups which have no nontrivial, finitely generated, normal subgroup of infinite index. Schreier showed in [4] that free groups are in $\mathcal{S}$. It is well-known that the fundamental groups of surfaces other than torus or Klein bottle are in $\mathcal{S}$, too. In the cases of torus and Klein bottle, the fundamental groups of them contain the infinite cyclic group as a nontrivial, finitely generated, normal subgroup of infinite index (see Proposition 15.23 in [6] for a proof). It is easy to see that both free groups and the fundamental groups of surfaces are either free products with amalgamation or HNN groups. According to Theorem 3.11 in [5], any free product with amalgamation $G=A *_{C} B$ with $C=\{1\}$ and $N \neq\{1\}$ also belongs to $\mathcal{S}$.

However, this result is not extended for groups of the form $A *_{C} B$ or $A *_{C}$, where both $A$ and $B$ are in $\mathcal{S}$ and $C$ is a free abelian group of finite rank. Let $G$ be the fundamental group of the trefoil knot complement in $S^{3}$. It is known that $G$ has the presentation $\left\langle a, b ; a^{2}=b^{3}\right\rangle$. Thus $G$ is of the form $A *_{C} B$, where $A, B$ and $C$ are infinite cyclic groups. Let $N_{1}=C$ and $N_{2}$ be the commutator subgroup of $G$. It can be easily shown that $N_{1}$ is normal in $G$ and $G / N_{1}$ is isomorphic to $\mathbb{Z}_{2} * \mathbb{Z}_{3}$. On the other hand, it can be easily observed that $N_{2}$ is the free group

Received by the editors September 5, 1997 and, in revised form, August 10, 1998.

1991 Mathematics Subject Classification. Primary 20E06, 30F40, 57M07.

Key words and phrases. Free product with amalgamation, HNN group, graph of groups, fundamental group, hyperbolic manifolds.

The author was partially supported by Konkuk University Research Fund and Korean Ministry of Education Research Fund, BSRI-98-1438. 
of rank 2 generated by $a b a^{-1} b-1$ and $a b^{2} a^{-1} b^{-2}$. Since $G / N_{2}$ is isomorphic to the first homology group of the trefoil knot complement in $S^{3}$, which is isomorphic to the infinite cyclic group, it follows that each of $N_{1}$ and $N_{2}$ is a finitely generated normal subgroup of infinite index. Hence $G$ is not in $\mathcal{S}$.

Let $G$ be a group of the form $A *_{C} B$ or $A *_{C}$, where $A, B \in \mathcal{S}$ and $C$ is a free abelian group of finite rank, and let $N$ be a nontrivial finitely generated normal subgroup of $G$. In this paper, we would like to give an answer to how $N$ behaves when $N$ fails to be of finite index in $G$. If $N$ is not of finite index in $G$, either $N$ is contained in $C$ like $N_{1}$ in the above example or $N$ intersects both $A$ and $B$ trivially like $N_{2}$ in the above example. Note that $N_{2} \cap A=\{1\}$ and $N_{2} \cap B=\{1\}$, as $G / N_{2}$ has no torsion element. In the latter case, $N$ turns out to be free. The result we obtain is the following theorem.

Theorem 2.1. Let $G=A *_{C} B$ or $G=A *_{C}$, where $C$ is a free abelian group of finite rank. Suppose that both $A$ and $B$ contain no finitely generated nontrivial normal subgroup of infinite index. If $N$ is a finitely generated normal subgroup of $G$ with $N$ not contained in $C$, then the index $|G: N|$ is finite or $N$ is free.

Ahlfors finiteness theorem implies that the fundamental group of a compact 3manifold, the interior of which admits a hyperbolic structure, is in the class $\mathcal{S}$ (see Theorem 3.1) if the manifold has at least one nontorus boundary. We derive Theorem 3.2 as an application of Theorem 2.1.

In $\S 1$ of this paper, we give a few technical lemmas on graphs of groups. In $\S 2$, we prove the main theorem, Theorem 2.1. In $\S 3$, we discuss an application of Theorem 2.1 to the fundamental group of a torus sum of two compact manifolds, each of which has at least one nontorus boundary component and admits a hyperbolic structure in its interior.

I would like to thank Peter Scott for introducing me to the ideas used in this paper. I also would like to thank the referee for helpful suggestions.

\section{Graph of Groups}

A graph of groups $(\Gamma, G)$ is defined to be a connected graph $\Gamma$ together with (a) groups $G_{v}$ and $G_{e}$ corresponding to each vertex $v$ of $\Gamma$ and edge $e$ of $\Gamma$, and (b) monomorphisms $\phi_{0}: G_{e} \rightarrow G_{v}$ and $\phi_{1}: G_{e} \rightarrow G_{w}$ for each edge $e$ of $\Gamma$, where $v$ and $w$ are the vertices of $e$.

Similarly, a graph of spaces $(\Gamma, X)$ consists of a connected graph $\Gamma$ together with (a) CW-complexes $X_{v}$ and $X_{e}$ corresponding to each vertex $v$ and edge $e$ of $\Gamma$, and (b) continuous maps $f_{0}: X_{e} \rightarrow X_{v}$ and $f_{1}: X_{e} \rightarrow X_{w}$ for each edge $e$ of $\Gamma$, where $v$ and $w$ are the vertices of $e$. Given a graph of spaces, we can define a total space $X_{\Gamma}$ as the quotient of

$$
\bigcup\left\{X_{v} \mid v \text { vertex of } \Gamma\right\} \cup \bigcup\left\{X_{e} \times I \mid e \text { edge of } \Gamma\right\}
$$

by the identifications

$$
\begin{aligned}
& X_{e} \times 0 \rightarrow X_{v} \quad \text { by } \quad(x, 0) \mapsto f_{0}(x), \\
& X_{e} \times 1 \rightarrow X_{w} \quad \text { by } \quad(x, 1) \mapsto f_{1}(x) .
\end{aligned}
$$

If $(\Gamma, X)$ is a graph of connected based spaces, then by taking fundamental groups, we obtain a graph of groups $(\Gamma, G)$ with the same underlying abstract graph $\Gamma$. The fundamental group of the graph of groups $(\Gamma, G)$ is defined to be the 


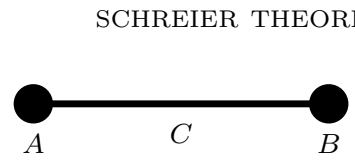

(a)

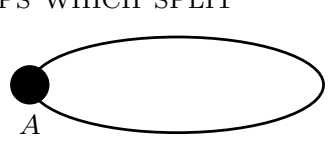

$C$

(b)

Figure 1. Free product with amalgamation and HNN group

fundamental group of the total space $X_{\Gamma}$. We denote by $G_{\Gamma}$ a graph of groups with fundamental group $G$ and underlying graph $\Gamma$.

For example, the free product with amalgamation $A *_{C} B$ is the fundamental group of the graph of groups in (a), Figure 1, where we have labelled the vertices and edge with the associated groups and $\phi_{0}: C \rightarrow A$ and $\phi_{1}: C \rightarrow B$ are the inclusions. In particular, if $G=A *_{C} B$ with $A \neq C \neq B$, we say that $G$ has nontrivial amalgamation.

The HNN group $A *_{C}$ is obtained by adjoining an element $t$ to $A$ subject to the relations $t^{-1} a t=\phi(a)$ for all $a \in A$, where $C$ is a subgroup of a group $G$ and $\phi: C \rightarrow A$ is a monomorphism. This group $A *_{C}$ can be considered as the fundamental group of the graph of groups of type (b) in Figure 1 when $\phi_{0}: C \rightarrow A$ is the inclusion map and $\phi_{1}: C \rightarrow A$ is the monomorphism $\phi$. For example, when $A=C=\mathbb{Z}, A *_{C}$ is the fundamental group of either torus (if $\phi: \mathbb{Z} \rightarrow \mathbb{Z}$ is the identity) or Klein bottle (if $\phi: \mathbb{Z} \rightarrow \mathbb{Z}$ is the isomorphism defined by $\phi(x)=-x$ ).

A group $G$ is said to split over a subgroup $C$ if either $G=A *_{C} B$ with $A \neq C \neq B$ or $G=A *_{C}$. If $G$ splits over some subgroup, we say that $G$ is splittable. For example, $\mathbb{Z}$ is splittable as $\mathbb{Z}=\{1\} *\{1\}$.

Let $H$ be a subgroup of $G$. Given a graph $G_{\Gamma}$ of groups, there is a graph of spaces $X_{\Gamma}$ corresponding to $G_{\Gamma}$. Consider the covering space $Y_{\Gamma_{1}}$ of $X_{\Gamma}$ whose vertex spaces and edge spaces are covering spaces of $X_{e}$ and $X_{v}$ for all edges $e$ and vertices $v$ of $\Gamma$ with $\pi_{1}\left(X_{\Gamma_{1}}\right)=H$. Using covering space theory, one can derive the following lemma (see [5] for a proof).

Lemma 1.1. If $G$ is the fundamental group of a graph $G_{\Gamma}$ of groups and $H<G$, then $H$ is the fundamental group of a graph of groups, where each vertex group is the intersection of $H$ and a conjugate of a vertex group of $G_{\Gamma}$ and each edge group is the intersection of $H$ and a conjugate of an edge group of $G_{\Gamma}$.

For convenience, we denote a conjugate $g^{-1} A g$ of $A$ by $A^{g}$.

Let $H_{\Gamma}$ be a graph of groups whose fundamental group $H$ is finitely generated. If $T$ is a maximal tree in $\Gamma, \Gamma \backslash T$ consists of finitely many edges as $H$ is finitely generated. Let $h_{1}, \cdots, h_{n}$ be generators of $H$. Each $h_{i}, i=1, \cdots, n$, is a product of a finite number of elements either belonging to vertex groups or corresponding to the edges in $\Gamma \backslash T$. Thus $h_{1}, \cdots, h_{n}$ are contained in a finite subgraph of groups of $H_{\Gamma}$. It follows that there is a finite subgraph of groups of $H_{\Gamma}$ which carries the whole fundamental group. Hence we obtain the following lemma.

Lemma 1.2. Let $H_{\Gamma}$ be a graph of groups whose fundamental group $H$ is finitely generated. Then there is a finite subgraph of groups of $H_{\Gamma}$ whose fundamental group is $H$.

We can define the distance between two vertices in a graph and the diameter of a graph. 
Definition 1.1. For any two vertices $P$ and $Q$ of a connected graph $\Gamma$, we define the distance $d(P, Q)$ between $P$ and $Q$ to be

$$
\min \{n \mid P \text { and } Q \text { are connected by } n \text { consecutive edges }\} \text {. }
$$

We say $\Gamma$ is of finite diameter if

$$
\sup \{d(P, Q) \mid P \text { and } Q \text { are vertices of } \Gamma\}
$$

is finite.

Suppose that $G$ splits over a subgroup $C$. For a graph of groups $G_{\Gamma}$ of type (a) or (b) in Figure 1, if the corresponding graph of groups to a normal subgroup of $G$ is of finite diameter, then it is in fact a finite graph of groups. In particular, we do not need the subgroup to be normal when $G$ is a free product with amalgamation.

Recall that a vertex $v$ in a graph is of valence 1 if $v$ has only one adjacent edge.

Lemma 1.3. Let $G=A *_{C} B$ with $A \neq C \neq B$. Suppose $G_{\Gamma}$ is a graph of groups of type (a) or (b) in Figure 1. If $H$ is a subgroup of $G$ and its corresponding graph of groups $H_{\Gamma_{1}}$ has finite diameter, then it is a finite graph of groups.

Proof. Let $X_{\Gamma}$ and $\tilde{X}_{\Gamma_{1}}$ be the graph of spaces corresponding to $G_{\Gamma}$ and $H_{\Gamma_{1}}$, respectively, so that $\tilde{X}_{\Gamma_{1}}$ is a covering space of $X_{\Gamma}$. First, we will show that there are only finitely many vertices of valence 1 in $H_{\Gamma_{1}}$. Let $\tilde{v}$ be a vertex of valence 1 in $H_{\Gamma_{1}}$ such that $H_{\tilde{v}}=H_{\tilde{e}}$ for the only edge $\tilde{e}$ having $\tilde{v}$ as its vertex. Let $\tilde{X}_{\tilde{v}}$ and $\tilde{X}_{\tilde{e}}$ be the vertex space and edge space corresponding to $H_{\tilde{v}}$ and $H_{\tilde{e}}$, respectively. The covering transformations fixing $\tilde{X}_{\tilde{v}}$ also fixes $\tilde{X}_{\tilde{e}}$ as $\tilde{v}$ is a vertex of valence 1 . So, in $X_{\Gamma}$, the fundamental group of one vertex space is the same as the fundamental group of the edge space. This implies that $A=C$ or $B=C$. This contradicts the hypothesis. Thus every vertex of valence 1 in $H_{\Gamma_{1}}$ yields a nontrivial amalgamation. Since $H$ is finitely generated, there are only finitely many vertices of valence 1 .

Suppose $\Gamma_{1}$ has an infinite number of vertices of valence greater than one. $\Gamma_{1}$ cannot have infinitely many loops as $H$ is finitely generated. By deleting a finite number of edges, we obtain a connected subtree $T$ of $\Gamma_{1}$ with infinitely many vertices, all but a finite number of which are of valence greater than 1 . Since all but a finite number of the vertices in $T$ has valence greater than 1 and $T$ is a tree, we can construct an infinite number of consecutive edges in $T$. This implies that $T$ has infinite diameter, and so $\Gamma_{1}$ has infinite diameter. This is a contradiction to the hypothesis. Hence there are only finitely many vertices of valence greater than 1.

Now we know that there are only finitely many vertices in $H_{\Gamma_{1}}$. Note that a graph having finitely many vertices and infinitely many edges must have infinitely many loops. This is impossible as $H$ is finitely generated. Therefore, $H_{\Gamma_{1}}$ is a finite graph.

Lemma 1.4. Let $G=A *_{C}$ and let $G_{\Gamma}$ be the corresponding graph of group of type (b) in Figure 1. If $N$ is a normal subgroup of $G$ and its corresponding graph of groups $N_{\Gamma_{1}}$ has finite diameter, then it is a finite graph of groups.

Proof. Let $X_{\Gamma}$ and $\tilde{X}_{\Gamma_{1}}$ be the graph of spaces corresponding to $G_{\Gamma}$ and $N_{\Gamma_{1}}$, respectively, so that $\tilde{X}_{\Gamma_{1}}$ is a covering space of $X_{\Gamma}$. We first consider the case where $\Gamma_{1}$ has a vertex of valence 1 . If there is a vertex $\tilde{v}$ of valence 1 in $N_{\Gamma_{1}}$, then every vertex in $N_{\Gamma_{1}}$ is of valence 1 as the covering is regular. Thus the only possible type 
for $\Gamma_{1}$ is the graph with two vertices and one edge connecting them. Hence $N_{\Gamma_{1}}$ is a finite graph of groups.

Now suppose all of the vertices in $\Gamma_{1}$ are of valence greater than 1 . By the same argument as in the proof of Lemma 1.3, we obtain a contradiction. Hence there are only finitely many vertices of valence 1 .

Since $\Gamma_{1}$ has a finite number of vertices and $H$ is finitely generated, $\Gamma_{1}$ is a finite graph.

For a finite graph of groups whose fundamental group is finitely generated, all vertex groups are finitely generated under the condition that all edge groups are finitely generated.

Lemma 1.5. Let $H$ be a finitely generated group. If $H_{\Gamma}$ is a finite graph of groups whose fundamental group is $H$, and if all edge groups of $H_{\Gamma}$ are finitely generated, then all vertex groups of $H_{\Gamma}$ are also finitely generated.

Proof. Let $H_{v}$ be an arbitrary vertex group of $H_{\Gamma}$. Since $\Gamma$ is finite, there are only finitely many edges which have $v$ as their common vertex. Let $e_{1}, \ldots, e_{k}$ be such edges. Let $V_{1}=\left\langle H_{e_{1}}, \ldots, H_{e_{k}}\right\rangle$. Assume $H_{v}$ is not finitely generated. Then there is an infinite sequence of finitely generated subgroups of $H_{v}$ such that

$$
V_{1} \subsetneq V_{2} \subsetneq V_{3} \subsetneq \ldots \text { and } \bigcup_{i=1}^{\infty} V_{i}=H_{v} .
$$

Since the $H_{e_{j}}$ 's are subgroups of $V_{i}$ 's, we can construct a graph $X_{i}$ of groups from $H_{\Gamma}$ by replacing $H_{v}$ with $V_{i}$. Set $W_{i}$ to be the fundamental group of $X_{i}$. Then $W_{i} \cap H_{v}=V_{i}$. Hence we have an infinite sequence $\left\{W_{i}\right\}$ of subgroups of $H$ such that

$$
W_{1} \subsetneq W_{2} \subsetneq W_{3} \subsetneq \ldots
$$

and $\bigcup_{i=1}^{\infty} W_{i}=H$. It follows that $H$ is not finitely generated, which is a contradiction. Therefore, $H_{v}$ is finitely generated.

\section{Groups SPlitting OVER Free ABElian Groups of Finite RANK}

Let $G$ be a group which splits over a group $C$. Suppose $G$ has a finitely generated normal subgroup $N$. Consider the graph of groups $G_{\Gamma}$ corresponding to $G$ of type either (a) or (b) in Figure 1. There is a corresponding graph of groups $N_{\Gamma_{1}}$ to $N$, so that the corresponding graph of spaces $Y_{\Gamma_{1}}$ to $N_{\Gamma_{1}}$ is a covering space of the graph of spaces $X_{\Gamma}$ corresponding to $G_{\Gamma}$. If $\Gamma_{1}$ is of infinite diameter, then it turns out that $N$ is contained in $C$.

Lemma 2.1. Let $G=A *_{C} B$ or $G=A *_{C}$ with a finitely generated normal subgroup $N$. If the corresponding graph of groups $N_{\Gamma_{1}}$ with vertex groups $\left\{N \cap A^{g}: g \in\right.$ $G\} \cup\left\{N \cap B^{g}: g \in G\right\}$ and edge groups $\left\{N \cap C^{g}: g \in G\right\}$ is of infinite diameter, then $N$ is contained in $C$.

Proof. If $\Gamma_{1}$ is of infinite diameter, then $\Gamma_{1}$ is a tree. In fact, if $\Gamma_{1}$ were not a tree, there would be an edge which is not in a maximal tree of $\Gamma_{1}$. Such an edge would be a part of a loop $L$. Since the covering is regular and $\Gamma_{1}$ is of infinite diameter, there is a covering transformation $g_{1}$ such that $g_{1} L \cap L=\emptyset$. By the same reason, there are an infinite number of covering transformations $g_{n}, n=1,2, \cdots$, such that $L, g_{1} L, g_{2} L, \cdots$ are mutually disjoint. Thus there would be an infinite number of 


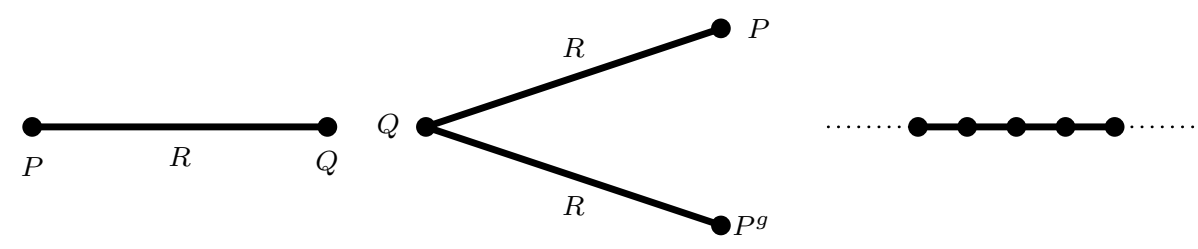

(a)

(b)

(c)

FigURE 2.

loops in $\Gamma_{1}$. This is a contradiction to the fact that $N$ is finitely generated. It follows that $\Gamma_{1}$ is a tree.

Since $N$ is finitely generated, there is a finite subgraph of groups $N_{\Gamma_{2}}$ of $N_{\Gamma_{1}}$ whose fundamental group is $N$. It follows that there is an infinite subtree of groups $T$ whose fundamental group is a vertex group. Also, in $T$, there is a tree of groups consisting of two vertex groups and one edge group and with trivial amalgamation, i.e., one of the vertex groups is the same as the edge group. Let $E$ be the graph of groups with two vertex groups $P$ and $Q$, and one edge group $R$ satisfying $Q=R$ as in (a), Figure 2. Since the covering is regular, every subgraph of groups with two vertex groups and one edge group is of the same type as $E$. We have two cases to consider.

In the case where $G=A *_{C} B, P$ should be the same as $R$. If not, we have a tree of groups of type (a) in Figure 2 with $P \neq R$, which produces a tree of groups of type (b) in Figure 2 with $P \neq R \neq P^{g}$ and $Q=R$. It is impossible to have a tree of groups of such type in $T$, as a vertex group of $T$ carries the whole fundamental group of $T$. Now, since $P=R=Q$, every vertex group in $N_{\Gamma_{1}}$ is an edge group. Hence the fundamental group $N$ of $N_{\Gamma_{1}}$ is an edge group $R$, which is a subgroup of $C$. It follows that $N$ is contained in $C$.

In the case where $G=A *_{C}, \Gamma_{1}$ should be a tree of type (c) in Figure 2 as $\Gamma_{1}$ is a tree and the covering is regular. If $P \neq R, N=\bigcup_{-\infty}^{\infty} N_{i}$, where each $N_{i}$ is a vertex group and $N_{i} \subsetneq N_{i+1}$. Since $N$ is finitely generated, it is impossible. Hence $P=R$, and so $N$ is an edge group. It follows that $N$ is contained in $C$.

Now we are in a position to prove the main theorem of this paper.

Theorem 2.1. Let $G=A *_{C} B$ or $G=A *_{C}$, where $C$ is a free abelian group of finite rank. Suppose that both $A$ and $B$ contain no finitely generated nontrivial normal subgroup of infinite index. If $N$ is a finitely generated normal subgroup of $G$ with $N$ not contained in $C$, then the index $|G: N|$ is finite or $N$ is free.

Proof. We will prove the theorem in the case where $G=A *_{C} B$. The case for $G=A *_{C}$ is the same. Let $G_{\Gamma}$ be the graph of groups with two vertex groups $A$ and $B$, and one edge group $C$. There is a corresponding graph of groups $N_{\Gamma_{1}}$ to $N$, so that the corresponding graph of spaces $Y_{\Gamma_{1}}$ to $N_{\Gamma_{1}}$ is a covering space of the graph of spaces $X_{\Gamma}$ corresponding to $G_{\Gamma}$. Since $N$ is not contained in $C$, Lemma 2.1 assures us that $N_{\Gamma_{1}}$ is of finite diameter. By Lemma 1.3 and Lemma 1.4, $N_{\Gamma_{1}}$ is a finite graph of groups. Since $C$ is a free abelian group of finite rank, all the 
edge groups of $N_{\Gamma_{1}}$ are finitely generated. By Lemma 1.5, all the vertex groups are finitely generated.

If both $N \cap A$ and $N \cap B$ are trivial, all the vertex groups and edges groups of $N_{\Gamma_{1}}$ are trivial. It follows that $N$ is free.

Now suppose that $N \cap A \neq\{1\}$ or $N \cap B \neq\{1\}$. Without loss of generality, we may assume that $N \cap A \neq\{1\}$. Then the index $|A: N \cap A|$ is finite, as $N \cap A$ is a finitely generated nontrivial normal subgroup of $A$. It follows that the covering is finite, as the covering is regular. Hence the index $|G: N|$ is finite.

\section{Application to Kleinian groups}

Recall that a Kleinian group is a discrete subgroup of $P S L_{2}(\mathbb{C})$, which is the group of isometries of $\mathbb{H}^{3}$, the 3-dimensional hyperbolic space. A hyperbolic 3manifold is defined to be a manifold which is the quotient of $\mathbb{H}^{3}$ by a torsion free Kleinian group $G$ acting as a covering group.

Thinking of $\mathbb{H}^{3}$ as the interior of the closed 3-ball $B^{3}$, we may think of $G$ as a group of conformal transformations of $S_{\infty}^{2}$, which is $\partial B^{3}$, the sphere at infinity. This action and the action on $H^{3}$ fit together to form an action on the closed ball $B^{3}$. The group $G$ acts properly on $\operatorname{Int}\left(B^{3}\right)$, but it does not act properly discontinuously on $S_{\infty}^{2}$. The limit set $L_{G}$ of $G$ is defined to be

$$
\left\{x \in S_{\infty}^{2} \mid g_{n}(y) \rightarrow x \quad \text { for some } y \in \mathbb{H}^{3} \text { and } g_{n} \in G\right\} .
$$

The complement of $L_{G}$ in $S_{\infty}^{2}$ is called the domain of discontinuity of $G$ and denoted by $D_{G}$.

The limit set of a Kleinian group contains either less than 3 elements or an infinite number of elements. A Kleinian group $G$ is called nonelementary if $L_{G}$ contains an infinite number of elements. It is known that if $N$ is a nontrivial normal subgroup of a nonelementary Kleinian group $G$, then $L_{G}=L_{N}$.

A Kleinian group $G$ is said to be of 2-nd kind if $L_{G}$ is not the whole sphere $S_{\infty}^{2}$.

Let $M$ be a compact 3-manifold which has at least one nontorus boundary component and hyperbolic structure in its interior. $M$ is homeomorphic to a 3-manifold $\left(\mathbb{H}^{3} \cup D_{G}\right) / G$ with the nonempty boundary $D_{G} / G$, where $G$ is a nonelementary Kleinian group of 2-nd kind (see [2] or 3]). Ahlfor's finiteness theorem says that if $G$ is a finitely generated discrete torsion-free Kleinian group, then $D_{G}$ is a finite collection of finite area hyperbolic surfaces (see [1]). This implies the following theorem.

Theorem 3.1. Let $G$ be the fundamental group of a compact 3-manifold $M$ which has a nontorus boundary component and hyperbolic structure in its interior. If $N$ is a nontrivial, finitely generated, normal subgroup of $G$, then the index $|G: N|$ is finite.

Proof. Since $N$ is normal in $G$ and $G$ is a nonelementary Kleinian group, $L_{N}=L_{G}$. It follows that $D_{G}=D_{N}$. Since $G$ is a Kleinian group of 2-nd kind, $D_{G} \neq \emptyset$. By the Ahlfors finiteness theorem, $D_{G} / G$ and $D_{N} / N$ are finite area hyperbolic surfaces. Hence the covering $D_{N} / N \rightarrow D_{G} / G$ must be finite. Therefore $N$ is of finite index in $G$.

Let $M$ be a torus sum of two compact 3-manifolds each of which has at least one nontorus boundary component and has hyperbolic structure in its interior. Then the fundamental group of $M$ is a free product with amalgamation $A *_{C} B$, where 
both $A$ and $B$ are nonelementary Kleinian groups of 2-nd kind and $C$ is a torus group. Theorem 3.1 and Theorem 2.1 produce the following theorem.

Theorem 3.2. Let $G$ be the fundamental group of a torus sum of two compact 3-manifolds each of which has at least one nontorus boundary component and has hyperbolic structure in its interior. If $N$ is a nontrivial, finitely generated, normal subgroup of $G$, then the index $|G: N|$ is finite or $N$ is free.

Proof. By Theorem 3.1, both $A$ and $B$ have no finitely generated normal subgroup of infinite index. If $N$ were in $C$, then $N$ would be a finitely generated normal subgroup of infinite index of $A$, which is impossible. Hence $N$ is not contained in $C$. By Theorem 2.1, $|G: N|$ is finite or $N$ is free.

\section{REFERENCES}

1. L. V. Ahlfors, Finitely generated Kleinian groups, Amer. J. Math. 86 (1964), 413-429. MR 29:4890

2. B. Maskit, Kleinian Groups, Springer-Verlag (1987), Berlin. MR 90a:30132

3. J. Morgan, Uniformization theorem for 3-manifolds, Smith Conjecture edited by J. Morgan and H. Bass, Academic Press (1984), 37-125. CMP 17:01

4. O. Schreier, Die Untergruppen der freien Gruppen, Abh. Math. Sem. Univ. Hamburg 5 (1928).

5. P. Scott and T. Wall, Topological methods in group theory, Homological group theory, London Math. Soc. Lecture Notes 36, Cambridge Univ. Press (1979), 137-203. MR 81m:57002

6. H. Zieschang, Finite groups of mapping classes of surfaces, Lecture notes in mathematics, Springer-Verlag, Berlin (1981). MR 86g:57001

Department of Mathematics Education, Konkuk University, Seoul 143-701, Korea

E-mail address: mhmoon@kkucc.konkuk.ac.kr 